\title{
Estimating Policy Position of the Candidates in the 2017 Gubernatorial Election in Jakarta
}

\author{
Aditya Batara Gunawan Universitas Bakrie, Indonesia
}

\begin{abstract}
This study attempts to understand the policy position of candidates in the 2017 gubernatorial election in Jakarta. The concept of policy position has been utilized in the electoral studies to distinguish policy orientation of political parties/candidates, and explaining voter preferences in electoral competition. Therefore, this study introduces the application of the quantitative text analysis method with the Wordfish model towards electoral platform of candidates in the 2017 gubernatorial election in Jakarta to estimate their policy positions. The model calculates words distribution and words frequency in the electoral platforms using simple Poisson process of statistical distribution. The process results in two key findings: two pairs of candidates (AgusSylvy and Anies-Sandi) indicate closer policy distance in the policy space, and socioeconomic issues emerge as central feature in the political rhetoric across the candidates. These findings are further confirmed by the observation of the voter preference distributions and the public statements of each candidate during the election.
\end{abstract}

Keywords:

Jakarta; Gubernatorial Election; Policy Position; Voter Preference; Quantitative Text Analysis

\section{INTRODUCTION}

The 2017 gubernatorial election in Jakarta has triggered debates among scholars and observers of Indonesian politics on the rise of sectarian politics in the largest Muslim-populous democracy in the world. As the capital of Indonesia, the politics of Jakarta's urban always been

\footnotetext{
Aditya Batara is a lecturer and researcher at Department International Relation-Universitas Bakrie, JI.H.R. Rasuna Said Kav C-22, Kuningan Jakarta Indonesia and also doctoral student at Political Science Institute in University of Heidelberg, Bergheimer Str. 58 D-69115 Germany. His research interest about military politics in Indonesia and peace and conflict resolution. (aditya.gunawan@bakrie.ac.id).

I am thankful to participants at the Prodipol International Symposium on Indonesian Politics April 2018 hosted by Universitas Negeri Semarang-Indonesia for their valuable comments on the early draft of this paper.
}

part of national mass media attention. But it receives significant attention when the capitol became the stepping stone for Joko 'Jokowi' Widodo, an outsider for Jakarta's elite circle, to gain widespread popularity when he was elected as the Governor of Jakarta in 2012 (Hamid, 2014). Two years later, Jokowi took part in the presidential election and got elected as the current president. The rise of Jokowi in a relatively short period has developed into sort of conventional wisdom widely held among politicians and commentators about Jakarta's strategic position: anyone who gets into power in the capitol administration holds 
the key to shape the future constellation of Indonesian politics. Such wisdom was clearly reflected in the 2017 Gubernatorial election in Jakarta that was followed by three pairs of high-profile candidates: Agus Yudhoyono (former military officers, son of former President Susilo Bambang Yudhoyono)-Sylviana Murni (high-level bureaucrats); Anies Baswedan (former Minister of Education in Jokowi administration)-Sandiaga Uno (businessman); and Basuki Tjahaja Purnama or well-known as "Ahok" (incumbent, former Joko Widodo's vice governor of Jakarta)-Djarot Saiful Hidayat (former Mayor of Blitar). In the second round of election, Anies defeated Basuki (Ahok) with slight margin and later, Ahok was sentenced by the court for blasphemy.

Central to the debate among scholars and observers is whether religious issue seems to be the underpinning factor that defines the outcome of Jakarta's 2017 election or not. Three different perspectives emerge in this context. Some scholars-and most of the mass media headlines-highlighted to the increasing influence of Islamic activism in Indonesian politics. The core argument is that religious polarization during the Jakarta election was orchestrated by the campaign managers, politicians, spin doctors, and the "conservative" Islamist groups (Ubaid \& Habibisubandi, 2017). The momentum for their cooperation was happened after the incumbent, Ahok, a Christian with ethnic Chinese background, made a statement about particular Quranic verse and the upcoming gubernatorial election. The statement was then accused of insulting the Quran and blasphemy, particularly by the conservative Islamists groups and several politicians. Series of demonstrations were organized by the groups, involving mass rallies in Jakarta streets. As anticipated, the protests changed into massive antiAhok campaign, urging Muslim population in Jakarta not to vote Ahok for the second-term and demanding Ahok to be brought into trial for blasphemy. Consequently, Ahok faced voters who preferred his policies but less likely to cast vote for him (Mietzner \& Muhtadi, 2017). The other perspective offers the salience of class for the Jakarta's 2017 election (Wilson, 2017). With its metropolitan status, Jakarta is among the eight provinces with highest Gini ratio (0.397) in 2016 (BPS, 2017). Instead of sectarian politics, high level income gap is viewed as the fundamental drive for Jakartans, which mostly living under poor 
condition in slump area, to cast their vote against the incumbent. Issues such as poverty (kemiskinan) and forced eviction (penggusuran) are considered prevalent among the poor Jakartans. Such argument is not overrated, considering that within two years of Ahok administration, more than 25.000 people become the victim of forced eviction, the highest record in the last decade (Kompas, 2017). For the urban poor, Ahok had broken his political promises in the 2012 election-with Jokowi-towards the Jakarta's urban poor to avoid forced eviction. And this condition was wellidentified by Anies-Sandi, further establishing political contract with the urban poor community that later contribute significantly to their vote share in the second round (Savirani \& Aspinall, 2017). Finally, other scholars attempts to bridge the aforementioned perspectives, arguing that the election was colored by interplay between religion and inequality statuses among the Jakartans (Warburton $\&$ Gammon, 2017). They propose that it is possible for sectarian voting behavior is channeling the perspective among Muslim poor voters that income inequality is the product of ethnic-Chinese domination on Indonesia's economy (Warburton \& Gammon, 2017).
The main purpose of this study is, however, not to confirm any position in the aforementioned debate a priori. Rather, this study attempts to enrich our understanding on the 2017 gubernatorial election in Jakarta through novel approach on policy position and quantitative text analysis. In order to do so, the study proceeds in several parts. The next section explains the method of quantitative text analysis and the utilization Wordfish model for the study. Subsequently the study discusses theoretical framework of policy position and election in the literature as well as the logic of applying Wordfish model as an approach. This part then followed by a section dedicated to elucidate the findings of text scaling process done with Wordfish model on the electoral platform of candidates in the Jakarta Election. Estimated policy position of candidate and list of political rhetoric used by the candidate are included in the section. Based on the findings, the next section provides observation on the nexus between policy position of candidates with the voter preference distribution, and the manifestation of political rhetoric from candidates during their campaign. Finally, the concluding part summarizes the findings and explains future potential of 
using quantitative text analysis for political studies in Indonesia.

\section{THEORETICAL PERSPECTIVES}

\section{Research Method}

This study applies quantitative text analysis method to estimate the policy position of each candidate during the 2017 gubernatorial election in Jakarta. Specifically, it utilizes word scaling algorithm called Wordfish (Slapin \& Proksch, 2008). Within the political science literature, the Wordfish model has been used by scholars to measure political position of parties in western/nonwestern democracies, legislators, interest groups, and presidents (Arnold, Doyle, \& Wiesehomeier, 2017; Aydogan \& Slapin, 2015; Klüver, 2009; Proksch \& Slapin, 2010). Principally, the model treats political documents as distinct political position of the political actors who produce it based on the word's usage and frequency. Using simple Poisson process of statistical distribution, the model calculates words frequencies from each political document with formulation as follows.

$$
\begin{aligned}
& y i j \sim \text { Poisson ( } \lambda i j) \\
& \lambda i j=\exp \left(\alpha i+\psi j+6 j{ }^{*} \omega i\right)
\end{aligned}
$$

Poisson distribution simply uses one parameter $\lambda$ which covers the mean and the variance. The count of word $j$ in electoral platform $i$ is yij. $\alpha$ is a set of text effects controlling the platform length. $\psi$ is a set of word fixed effects that control for the fact that some words generally used more frequently than other words by all candidate, for example conjunctions, preposition, and so on. Meanwhile, $\beta$ is an estimate of a word-specific weight showing the importance of word $j$ in discriminating between policy positions and $\omega$ is the estimate of platform/candidate is policy position. Expectation maximization (EM) algorithm is repeatedly applied between these components to locate the position of candidate.

The application of Wordfish model in this paper follows several steps. First, the political documents are the electoral platform/official campaign documents (dokumen visi dan misı) from each pair of the candidates that had been submitted to the election commission (KPUD DKI Jakarta, Komisi Pemilihan Umum Daerah DKI Jakarta) before the campaign period and the Election Day. These electoral platforms are accessible from the url: https://kpujakarta.go.id/download/. All of the documents then converted into text format, resulting in total of 8.535 words. 
In the second step, the text data is transformed into matrix of word frequencies format. The matrix comprises two key features: document identity and words as variable. Several rules applied when processing the data such as fixing typos and removing names of candidate, numbers, points/bullets, dots, comma, or parentheses. Finally, the third step is to run the Wordfish model in the $\mathrm{R}$ statistical language. ${ }^{1}$ During the process, the word frequency matrix is processed through writing the Wordfish code in $\mathrm{R}$ to run the algorithm. The output is candidate position with standard error, word weight, and word fixed-effects. Based on the calculation of policy position and word distributions for each candidate, this study then observes the voter preferences distribution and manifestation of respective word in the candidate campaign rhetoric qualitatively.

\section{LITERATURE REVIEW}

Studies on sub-national politics in Indonesia are flourishing after the implementation of direct election (Pilkada) for sub-national leader in 2005 (Choi, 2011; Erb \& Sulistiyanto, 2009; Hadiz,

${ }^{1} \mathrm{R}$ is a free software environment for statistical computing and graphics that can be downloaded from https://www.r-project.org/. This study applies the Wordfish code implemented in the Austin package (Lowe, 2015).
2010; Klinken, 2009; Schulte Nordholt \& Klinken, 2007). Most of these studies emphasize 'informal politics' in the form of clientelism or political dynasty (family relationship) to explain election dynamics and outcome. It seems that the logic behind the sub-national politics research is influenced by the knowledge produced on the study of Indonesian political party. Study on Indonesian political party highlights post-Suharto political parties as weakly institutionalized and indicates the waning of ideological stand of each party (Ambardi, 2009; Mietzner, 2013; Slater \& Simmons, 2013). Consequently, candidates running at the sub-national level extensively depend on extra-party strategy to win the voters such as family networks, patronage, vote buying (giving money or goods), or clientelism (promising jobs and allocating government contracts in return for support) directly to potential voters (Allen, 2015; Aspinall \& As'ad, 2016; Aspinall \& Sukmajati, 2016; Berenschot, 2018; Sobari, 2018). Other study offers another alternative on Indonesia's local politics. It finds that voters in three major cities also utilize retrospective reasoning when casting their vote, willing to spend their time to acquire information about the incumbent performance and other 
candidate's background (Fossati, 2018). Nevertheless, it also pointed out that partisanship of the voters remains less significant in the process.

If party in Indonesia is weakly institutionalized and becoming less polarized ideologically, then what differentiate one candidate to another in electoral competition? Principally this has been the central theme in electoral studies. Politics levitate around the ability to exercise power, which holds farreaching consequence on people's life. If one can control the legitimate authority then she/he can make policies that serve his own political interest or his constituency needs, thus improving opportunity for re-election. Taking this view into consideration, policy position then emerges as the important concept that can be used for making sense of candidate differences during the election. It is through policy position of each candidate that voters are able to decide their vote for certain candidate. For the candidates, this means that they must work with programmatic politics to win the heart of voters. And this type of strategy was evident in Indonesia, as one study found during the 2017 election in Kulon Progo district (Mas'udi \& Kurniawan, 2017). The policy position concept was rooted in the seminal work "An Economic Theory of Democracy" by Anthony Downs (1957). The core is that voters will more likely to vote political party/candidate with closest policy position to their own (Tomz \& Van Houweling, 2008). Put simply, if a voter favors the improvement of public service, then it is more likely for her/him to vote candidate who promote or promise to improve public service. In short, mechanism of voting behavior is largely defined by the proximity of voter's position and the candidate position on certain issues/context. In the election, Downs proposes that "each of them [voters] views elections strictly as means of selecting the government most beneficial to him. Each citizen estimates the utility income from government action he expects each party would provide him if it were in power in the forthcoming election period (Downs 1957b, p. 138).' Hence, voters are rational actors: they are able to locate political actor in a single continuum of possible policy alternatives and make their own choice (Adams \& Meril III, 2005). The rationality of voters is manifested by the existence of transitive preferences i.e. if the voter prefers candidate $A$ over candidate $B$ and candidate $B$ is preferred over candidate $C$, then candidate $A$ is 
preferred by the voter over candidate $C$ literature: hand-coding (CMP, $(A>B>C=A>C)$. The core assumption is comparative manifesto project), and simple; voter holds rank order preferences computer-assisted content analysis in order and rarely violate it. Empirically, (Wordscores or Wordfish) (Benoit \& Laver, this preference order can be observed 2003; Klingemann, Volkens, Bara, through surveys of voter preference on Budge, \& McDonald, 2006; Laver, candidate prior and after the election (exit Benoit, \& Gary, 2003; Slapin \& Proksch, poll).

The subsequent issue is to implement feasible method in measuring policy 2008). All of these techniques have position. Scholars of political party depend on three sources of data when dealing with estimating policy position: expert surveys, voter survey, legislator survey, and text (Laver, 2014). Of course, each type of data has different degree of advantage and most importantly, depending on the research objective. Using legislator survey, for example, runs the risk of greater bias since politicians are able to manipulate their policy position for strategic purpose. Meanwhile, expert survey and voter survey demand enormous resources financially. Based on these considerations, this study decides to use text-electoral platforms of candidatesince it is the most accessible source of data. Furthermore, recent technique of quantitative text analysis is able to address the issue of validity in textual data. There are three main techniques for extracting policy position in the existing similarity: utilizing text not as discourse that need to be interpreted or explained, but rather as data in forms of words. Nevertheless, one need to be careful when deciding which technique fits the research objective. The hand-coding technique and Wordscores demand a priori definition of policy dimension. Thus, researcher needs to define which political document/text can be referred as left or right on the ideological spectrum of a polity. Unlike the formers, the Wordfish model does not require any reference document to measure the policy distance. The Wordfish model assumes that the relative use of words by political actors (political party, legislators, president, electoral candidate, and even interest groups) could provide information about their position in a policy space/dimension as defined by the documents (Arnold et al., 2017; Klüver, 2009; Proksch \& Slapin, 2010; Slapin \& Proksch, 2008). This is particularly important when the 
Figure 1. Policy position of the candidate in the Jakarta's 2017 Gubernatorial Election

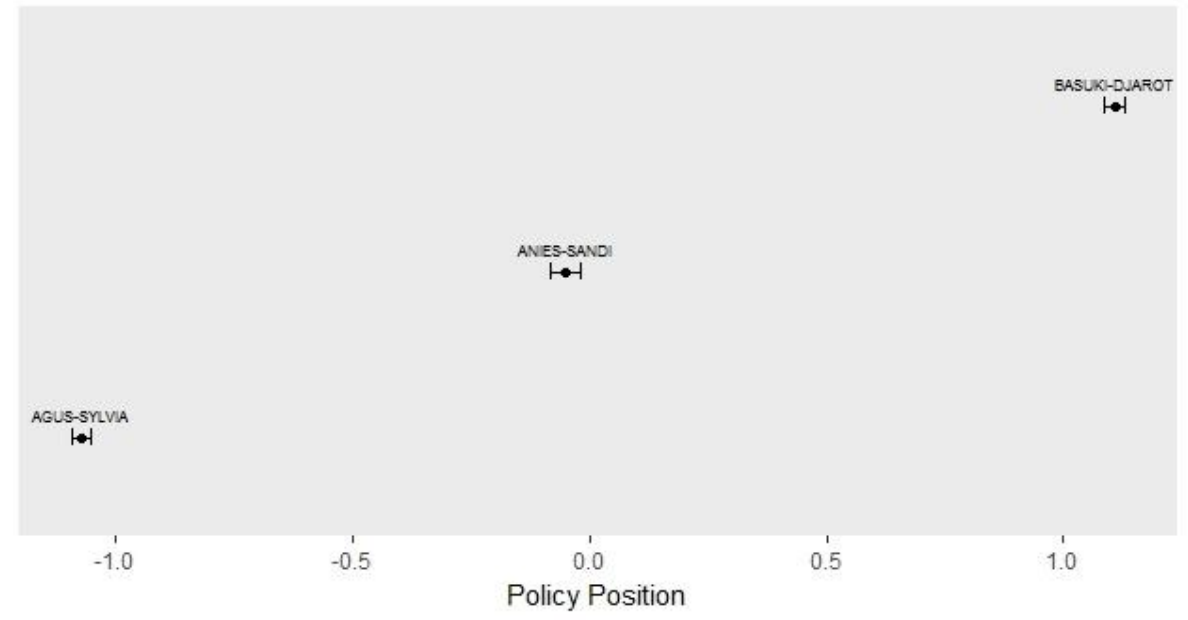

left-right spectrum of ideological leaning interpret policy position of political actors such as in Western Europe is non-existent in post-Suharto Indonesia must be (Proksch, Slapin, \& Thies, 2011). And defined according to the empirical context similar condition fits well with the rather than a priori.

Indonesian case. In post-Suharto

In essence, the novelty of this study Indonesia, the left politics is virtually nonrests on the application of policy position existent. Above all, communist and/or any concept and its measurement technique leftist ideologies remains forbidden in to understand the dynamic of subIndonesia, making it less likely for a leftist national election and voting behavior in party to compete in elections since 1966 contemporary Indonesia. Unlike the or establish strong support in the society. dominant 'informal politics' explanation of The latter condition was evident in the pilkada, this study departs from the failure of several left-leaning parties to assumption that voter's preference and secure parliamentary seats (Mietzner, coalition potential in the election can be 2013). In addition, the economic explained from the policy orientation platforms of many parties appeared to be preferred by the candidates. And such uniform, as all stressed their commitment objective can be achieved by using the to improving welfare among the lower- recent technique of measuring policy class and promulgated a protectionist position, which not necessitates stand (Liddle \& Mujani, 2007; Ufen, researcher to set an ideological parameter 2008). This means that any attempt to for the policy space. 
Table 1. Wordfish model calculation of candidate position in the 2017 Gubernatorial Election in Jakarta

\begin{tabular}{lcc}
\multicolumn{1}{c}{ Candidate } & Score & Standard Error \\
Agus-Sylvia & -1.07092 & 0.020098 \\
Anies-Sandi & -0.05045 & 0.031536 \\
Basuki-Djarot & 1.108655 & 0.021325
\end{tabular}

The Document-scaling Result of the 2017 emphasis on specific words that latter Gubernatorial Election differentiate their position in the policy

The Wordfish model locates Agus- scale. This also serves as the internal Sylvia and Basuki-Djarot at the end of validity of the model. To calculate words each continuum of policy scale (Figure.1). weight, the model establishes so-called Meanwhile, Anies-Sandi is located at the word-fixed effects i.e. frequency of slightly middle position of policy scale mentioned words. Words that frequently leaning towards Agus-Sylvia position. The mentioned (e.g. conjunctions, articles, numerical measure for each position and prepositions, etc.) that do not discriminate standard error of each candidate is between candidate supposed to have presented in Table 1.

The next important result is word 2008). In contrary, there are some words distribution among the candidates. As that used less frequent in the document aforementioned, Wordfish calculates and having smaller fixed effects. These words weight from the documents, are the politically-relevant language that meaning that each document put more differentiates the political actors in the

Figure 2. Word-fixed effects and word weights in the electoral platform of Jakarta election

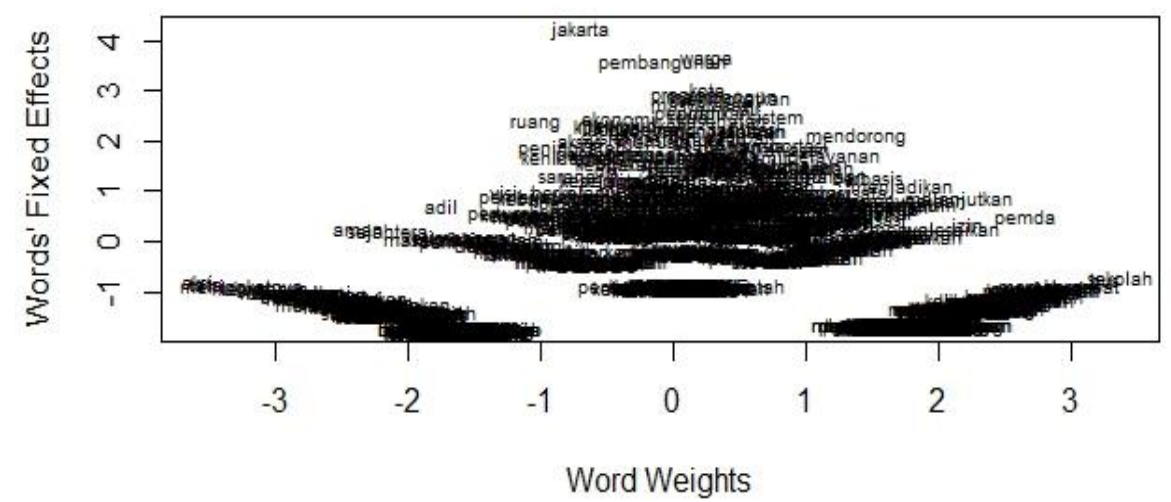


policy scale (Aydogan \& Slapin, 2015). In

this regard, words with a high fixed effect

have zero weight, but words with low

fixed effects have either negative or

positive weight (Slapin \& Proksch, 2008,

p. 715). Consequently, we will see an

Eiffel Tower plot from the model (see the

Figure 2).
In addition, the Wordfish model allows us to establish list of words association with the policy position of political actors. Thus, we may identify the political rhetoric emphasized by each candidate in the Jakarta election.

Table 2. Words associated with the left position of the scale

\begin{tabular}{|c|c|c|c|c|}
\hline $\begin{array}{l}\text { Demokrasi } \\
\text { (democracy) }\end{array}$ & $\begin{array}{l}\text { Historis } \\
\text { (historical) }\end{array}$ & Penindasan (oppression) & $\begin{array}{l}\text { Permasalahan } \\
\text { (problem) }\end{array}$ & Tingkat (level) \\
\hline Kerangka (framework) & Sentral (central) & Ketidakadilan (injustice) & Hak (right) & $\begin{array}{l}\text { Pemukiman } \\
\text { (settlement) }\end{array}$ \\
\hline Martabat (dignity) & Citra (image) & Takut (fear) & Muda (young) & Dasar (basic) \\
\hline Rakyat (people) & $\begin{array}{l}\text { Trans-negara } \\
\text { (trans-state) }\end{array}$ & $\begin{array}{l}\text { Ketidakpedulian } \\
\text { (uncaring) }\end{array}$ & $\begin{array}{l}\text { Keamanan } \\
\text { (security) }\end{array}$ & $\begin{array}{l}\text { Penyalahgunaan } \\
\text { (abuse) }\end{array}$ \\
\hline Pilihan (choice) & Sumber (source) & Manfaat (benefit) & $\begin{array}{l}\text { Pertumbuhan } \\
\text { (growth) }\end{array}$ & $\begin{array}{l}\text { Pemanfaatan } \\
\text { (utilization) }\end{array}$ \\
\hline Nilai (value) & Risiko (risk) & Social (social) & $\begin{array}{l}\text { Pemerintah } \\
\text { (government) }\end{array}$ & Green (green) \\
\hline Generasi (generation) & Lemah (weak) & Alam (nature) & Hijau (green) & Sipil (civil) \\
\hline $\begin{array}{l}\text { Megatrends(mega- } \\
\text { trends) }\end{array}$ & Dana (fund) & Dampak (impact) & Pusat (center) & $\begin{array}{l}\text { Pembinaan } \\
\text { (fostering) }\end{array}$ \\
\hline Revolusi (revolution) & $\begin{array}{l}\text { Non-budgeter } \\
\text { (non-budgetary) }\end{array}$ & Demografi (demography) & Nyata (existent) & $\begin{array}{l}\text { Efektivitas } \\
\text { (effectiveness) }\end{array}$ \\
\hline Bangsa (nation) & $\begin{array}{l}\text { Perijinan } \\
\text { (permit) }\end{array}$ & Arus (stream) & $\begin{array}{l}\text { Aparat } \\
\text { (apparatus) }\end{array}$ & $\begin{array}{l}\text { Kewirausahaan } \\
\text { (entrepreneurship) }\end{array}$ \\
\hline Pergeseran (shifting) & $\begin{array}{l}\text { Mahal } \\
\text { (expensive) }\end{array}$ & Perserikatan (union) & Taman (park) & $\begin{array}{l}\text { Lingkungan } \\
\text { (environment) }\end{array}$ \\
\hline $\begin{array}{l}\text { Geo-politik (geo- } \\
\text { politics) }\end{array}$ & $\begin{array}{l}\text { Provinsi } \\
\text { (province) }\end{array}$ & Pulau (island) & $\begin{array}{l}\text { Manajemen } \\
\text { (management) }\end{array}$ & $\begin{array}{l}\text { Hubungan } \\
\text { (relationship) }\end{array}$ \\
\hline $\begin{array}{l}\text { Geo-ekonomi (geo- } \\
\text { economy) }\end{array}$ & With (with) & Keterbukaan (openness) & $\begin{array}{l}\text { Kebahagiaan } \\
\text { (happiness) }\end{array}$ & Budaya (culture) \\
\hline Luhur (noble) & Equity (equity) & Sekat (partition) & Hidup (life) & Lintas (inter-) \\
\hline Tugas (duty) & $\begin{array}{l}\text { Kekinian } \\
\text { (contemporary) }\end{array}$ & Negara (state) & $\begin{array}{l}\text { Sarana } \\
\text { (medium) }\end{array}$ & Global (global) \\
\hline Andal (capable) & $\begin{array}{l}\text { Struktural } \\
\text { (structural) }\end{array}$ & $\begin{array}{l}\text { Konektivitas } \\
\text { (connectivity) }\end{array}$ & $\begin{array}{l}\text { Kriminalitas } \\
\text { (criminality) }\end{array}$ & $\begin{array}{l}\text { Keterlibatan } \\
\text { (involvement) }\end{array}$ \\
\hline Fasilitas (facility) & $\begin{array}{l}\text { Koordinasi } \\
\text { (coordination) }\end{array}$ & $\begin{array}{l}\text { Subordinasi } \\
\text { (subordination) }\end{array}$ & $\begin{array}{l}\text { Perkembangan } \\
\text { (progress) }\end{array}$ & $\begin{array}{l}\text { Kerjasama } \\
\text { (cooperation) }\end{array}$ \\
\hline Kreatif (creative) & $\begin{array}{l}\text { Optimistis } \\
\text { (optimistic) }\end{array}$ & Aspek (aspect) & $\begin{array}{l}\text { Peningkatan } \\
\text { (improvement) }\end{array}$ & $\begin{array}{l}\text { Umkm (abbv. Micro } \\
\text { and middle } \\
\text { enterprises) }\end{array}$ \\
\hline Jajaran (line) & $\begin{array}{l}\text { Kalangan } \\
\text { (group/class) }\end{array}$ & Rinci (detail) & Akses (access) & $\begin{array}{l}\text { Penataan } \\
\text { (regulating) }\end{array}$ \\
\hline $\begin{array}{l}\text { Kebersamaan } \\
\text { (togetherness) }\end{array}$ & $\begin{array}{l}\text { Prasana } \\
\text { (infrastructure) }\end{array}$ & Tajam (sharp) & $\begin{array}{l}\text { Perjuangan } \\
\text { (strugg/e) }\end{array}$ & Mobilitas(mobility) \\
\hline $\begin{array}{l}\text { Perwujudan } \\
\text { (realization) }\end{array}$ & $\begin{array}{l}\text { Kekuatan } \\
\text { (power) }\end{array}$ & Internet (internet) & $\begin{array}{l}\text { Keadilan } \\
\text { (justice) }\end{array}$ & Good (good) \\
\hline Adil (justice) & $\begin{array}{l}\text { Harmoni } \\
\text { (harmony) }\end{array}$ & Drainase (drainage) & $\begin{array}{l}\text { Dinamis } \\
\text { (dynamic) }\end{array}$ & Manusia (human) \\
\hline Harapan (hope) & $\begin{array}{l}\text { Toleransi } \\
\text { (tolerance) }\end{array}$ & Bantaran (flood plain) & Gini (gini) & Sanitasi (sanitation) \\
\hline
\end{tabular}




\begin{tabular}{|c|c|c|c|c|}
\hline Pertahanan (defense) & $\begin{array}{l}\text { Golongan } \\
\text { (group/class) }\end{array}$ & Premanisme (gangster) & Rasio (ratio) & $\begin{array}{l}\text { Pembangunan } \\
\text { (development) }\end{array}$ \\
\hline Perputaran (rotation) & $\begin{array}{l}\text { Kohesi } \\
\text { (cohesion) }\end{array}$ & Keterpaduan (solidity) & $\begin{array}{l}\text { Komunikasi } \\
\text { (communication) }\end{array}$ & Nasional (national) \\
\hline $\begin{array}{l}\text { Akumulasi } \\
\text { (accumulation) }\end{array}$ & $\begin{array}{l}\text { Keterampilan } \\
\text { (skill) }\end{array}$ & $\begin{array}{l}\text { Babinkamtibnas (non- } \\
\text { commissioned police } \\
\text { officer) }\end{array}$ & Isu (issue) & $\begin{array}{l}\text { Infrastruktur } \\
\text { (infrastructure) }\end{array}$ \\
\hline Kapital (capital) & $\begin{array}{l}\text { Sinergitas } \\
\text { (synergy) }\end{array}$ & $\begin{array}{l}\text { Babinsa (non- } \\
\text { commissioned military } \\
\text { officer) }\end{array}$ & $\begin{array}{l}\text { Rekreasi } \\
\text { (recreation) }\end{array}$ & Pekerjaan (job) \\
\hline Gedung (building) & Pola (pattern) & Monitoring (monitoring) & $\begin{array}{l}\text { Interaksi } \\
\text { (interaction) }\end{array}$ & Pantai (beach) \\
\hline Memori (memory) & $\begin{array}{l}\text { Unsur } \\
\text { (component) }\end{array}$ & Serangan (attack) & $\begin{array}{l}\text { Efisien } \\
\text { (efficiency) }\end{array}$ & \\
\hline Sejarah (history) & $\begin{array}{l}\text { Pemahaman } \\
\text { (understanding) }\end{array}$ & Teroris (terrorist) & $\begin{array}{l}\text { Kelompok } \\
\text { (group) }\end{array}$ & \\
\hline $\begin{array}{l}\text { Bodetabek (bogor, } \\
\text { depok, tangerang, } \\
\text { bekasi) }\end{array}$ & $\begin{array}{l}\text { Kesadaran } \\
\text { (awareness) }\end{array}$ & Penyebaran (spreading) & $\begin{array}{l}\text { Aspirasi } \\
\text { (aspiration) }\end{array}$ & \\
\hline Ruang (space) & Etika (ethics) & Kemiskinan (poverty) & Fakta (fact) & \\
\hline Kenyataan (reality) & $\begin{array}{l}\text { Pranata } \\
\text { (institution) }\end{array}$ & $\begin{array}{l}\text { Ketimpangan } \\
\text { (unbalanced) }\end{array}$ & $\begin{array}{l}\text { Kesenjangan } \\
\text { (discrepancy) }\end{array}$ & \\
\hline Sulit (difficult) & Public (public) & Shelter (shelter) & $\begin{array}{l}\text { Partnership } \\
\text { (partnership) }\end{array}$ & \\
\hline $\begin{array}{l}\text { Persentase } \\
\text { (percentage) }\end{array}$ & Private (private) & Optimum (optimum) & $\begin{array}{l}\text { Sustainable } \\
\text { (sustainable) }\end{array}$ & \\
\hline Kondisi (condition) & Growth (growth) & $\begin{array}{l}\text { Posyandu (integrated } \\
\text { health post) }\end{array}$ & $\begin{array}{l}\text { Alternatif } \\
\text { (alternative) }\end{array}$ & \\
\hline Jurang (gap) & $\begin{array}{l}\text { Ketidakamanan } \\
\text { (insecurity) }\end{array}$ & Kawasan (region) & $\begin{array}{l}\text { Uud } \\
\text { (constitution) }\end{array}$ & \\
\hline Kaya (rich) & $\begin{array}{l}\text { Kemampuan } \\
\text { (capability) }\end{array}$ & Heritage (heritage) & $\begin{array}{l}\text { Pemenuhan } \\
\text { (fulfillment) }\end{array}$ & \\
\hline Serius (serious) & $\begin{array}{l}\text { Pengalokasian } \\
\text { (allocation) }\end{array}$ & Ibadah (worship) & $\begin{array}{l}\text { Informal } \\
\text { (informal) }\end{array}$ & \\
\hline Metropolis (metropolis) & Mikro (micro) & Lahan (land) & Urban (urban) & \\
\hline Peredaran (circulation) & $\begin{array}{l}\text { Inisiatif } \\
\text { (initiative) }\end{array}$ & Wahana (mode) & $\begin{array}{l}\text { Farming } \\
\text { (farming) }\end{array}$ & \\
\hline $\begin{array}{l}\text { Keterasingan } \\
\text { (alienation) }\end{array}$ & $\begin{array}{l}\text { Pengintegrasian } \\
\text { (integrating) }\end{array}$ & Sepeda (bicycle) & Anak (children) & \\
\hline Jalanan (street) & $\begin{array}{l}\text { Kelestarian } \\
\text { (conservation) }\end{array}$ & Feeder (feeder) & $\begin{array}{l}\text { Tujuan } \\
\text { (objective) }\end{array}$ & \\
\hline Kepantasan (proper) & Kokoh (tough) & Centre (center) & $\begin{array}{l}\text { Nyaman } \\
\text { (comfort) }\end{array}$ & \\
\hline $\begin{array}{l}\text { Peradaban } \\
\text { (civilization) }\end{array}$ & Tertib (order) & Konseling (counselling) & $\begin{array}{l}\text { Produktif } \\
\text { (productive) }\end{array}$ & \\
\hline $\begin{array}{l}\text { Megapolitan } \\
\text { (megapolitan) }\end{array}$ & $\begin{array}{l}\text { Keragaman } \\
\text { (diversity) }\end{array}$ & Responsif (responsive) & Misi (mission) & \\
\hline Bukti (evidence) & Suku (tribe) & $\begin{array}{l}\text { Accountable } \\
\text { accountable) }\end{array}$ & $\begin{array}{l}\text { Dukungan } \\
\text { (support) }\end{array}$ & \\
\hline Model (model) & Agama (religion) & Aktual (actual) & Sosial (social) & \\
\hline Kebudayaan (culture) & $\begin{array}{l}\text { Sumberdaya } \\
\text { (resource) }\end{array}$ & Realistis (realistic) & Kualitas(quality) & \\
\hline $\begin{array}{l}\text { Homogenisasi } \\
\text { (homogenization) }\end{array}$ & Landasan (base) & Visioner (visionary) & Modal (capital) & \\
\hline $\begin{array}{l}\text { Kemajemukan } \\
\text { (plurality) }\end{array}$ & $\begin{array}{l}\text { Pancasila } \\
\text { (pancasila) }\end{array}$ & Elemen (element) & $\begin{array}{l}\text { Pemberdayaan } \\
\text { (empowerment) }\end{array}$ & \\
\hline Akut (acute) & $\begin{array}{l}\text { Orientasi } \\
\text { (orientation) }\end{array}$ & Komitmen (commitment) & $\begin{array}{l}\text { Kebijakan } \\
\text { (policy) }\end{array}$ & \\
\hline Buruk (bad) & Etis (ethical) & Kampanye (campaign) & $\begin{array}{l}\text { Ekonomi } \\
\text { (economy) }\end{array}$ & \\
\hline Suara (voice) & $\begin{array}{l}\text { Penghargaan } \\
\text { (award) }\end{array}$ & Operasional (operational) & $\begin{array}{l}\text { Kesejahteraan } \\
\text { (prosperity) }\end{array}$ & \\
\hline
\end{tabular}




\begin{tabular}{|c|c|c|c|}
\hline Pelaku (actor) & $\begin{array}{l}\text { Ekspresi } \\
\text { (expression) }\end{array}$ & Sasaran (target) & $\begin{array}{l}\text { Penyerapan } \\
\text { (absorption) }\end{array}$ \\
\hline Minim (minimum) & $\begin{array}{l}\text { Otentik } \\
\text { (authentic) }\end{array}$ & Mandat (mandate) & Arah (direction) \\
\hline Mental (mental) & $\begin{array}{l}\text { Belenggu } \\
\text { (handcuff) }\end{array}$ & Anggaran (budget) & Luas (wide) \\
\hline $\begin{array}{l}\text { Penggusuran (forced } \\
\text { eviction) }\end{array}$ & $\begin{array}{l}\text { Jangkauan } \\
\text { (extent) }\end{array}$ & Situasi (situation) & Pengguna (user) \\
\hline Restorasi (restoration) & $\begin{array}{l}\text { Kebebasan } \\
\text { (freedom) }\end{array}$ & Penghormatan (respect) & $\begin{array}{l}\text { Kepemimpinan } \\
\text { (leadership) }\end{array}$ \\
\hline Situs (site) & $\begin{array}{l}\text { Keterbelakangan } \\
\text { (backwardness) }\end{array}$ & Konflik (conflict) & Bersih (clean) \\
\hline
\end{tabular}

Note: The word list was obtained from the top 300 words that have the highest word weight. The verbs are dropped from the list since they have no ideological meaning. Similar words with different suffixes also dropped and only the root words preserved (see also, Aydogan \& Slapin, 2015).

Table 3. Words associated with the right position of the scale

\begin{tabular}{|c|c|c|c|c|}
\hline $\begin{array}{l}\text { Kebangsaan } \\
\text { (nationality) }\end{array}$ & $\begin{array}{l}\text { Finansial } \\
\text { (financial) }\end{array}$ & $\begin{array}{l}\text { Jabodetabek (jakarta- } \\
\text { bogor-depok-tangerang- } \\
\text { bekasi) }\end{array}$ & Waste (waste) & Tarik (pull) \\
\hline Abai (ignorant) & $\begin{array}{l}\text { Pemilihan } \\
\text { (election) }\end{array}$ & Jalur (route) & $\begin{array}{l}\text { Rusunawa (public } \\
\text { housing) }\end{array}$ & Bahari (maritime) \\
\hline Potensi (potential) & Badan (body) & Flyover (flyover) & Regulasi (regulation) & $\begin{array}{l}\text { Pkl (street } \\
\text { vendor) }\end{array}$ \\
\hline $\begin{array}{l}\text { Perpecahan } \\
\text { (disunity) }\end{array}$ & Gizi (nutrition) & Underpass (underpass) & Volume (volume) & Betawi (betawi) \\
\hline Entreprenuership & Daging (meat) & Ratio (ratio) & $\begin{array}{l}\text { Komunal } \\
\text { (communal) }\end{array}$ & $\begin{array}{l}\text { Pembuangan } \\
\text { (disposal) }\end{array}$ \\
\hline Kolusi (collusion) & $\begin{array}{l}\text { Ekosistem } \\
\text { (ecosystem) }\end{array}$ & Perlintasan (crossing) & Aturan (rule) & Bbg (gas fuel) \\
\hline $\begin{array}{l}\text { Nepotisme } \\
\text { (nepotism) }\end{array}$ & Kredit (credit) & Optimalisasi (optimization) & $\begin{array}{l}\text { Pembakaran } \\
\text { (combustion) }\end{array}$ & $\begin{array}{l}\text { Izin } \\
\text { (permitllicense) }\end{array}$ \\
\hline Toleran (tolerant) & $\begin{array}{l}\text { Pedagang } \\
\text { (traders) }\end{array}$ & $\begin{array}{l}\text { Penyempurnaan } \\
\text { (perfection) }\end{array}$ & $\begin{array}{l}\text { Larangan } \\
\text { (prohibition) }\end{array}$ & Rapi (tidy) \\
\hline $\begin{array}{l}\text { Partisipatif } \\
\text { (participative) }\end{array}$ & $\begin{array}{l}\text { Peternakan } \\
\text { (farming) }\end{array}$ & Command (command) & $\begin{array}{l}\text { Perda (provincial } \\
\text { rule) }\end{array}$ & Pangan (food) \\
\hline $\begin{array}{l}\text { Birokasi } \\
\text { (bureaucracy) }\end{array}$ & Hasil (result) & Komersial (commercial) & Penyediaan (supply) & $\begin{array}{l}\text { Keberlangsungan } \\
\text { (sustainability) }\end{array}$ \\
\hline Akta (certificate) & $\begin{array}{l}\text { Koperasi } \\
\text { (cooperative) }\end{array}$ & Transaksi (transaction) & Korupsi (corruption) & $\begin{array}{l}\text { Institusi } \\
\text { (institution) }\end{array}$ \\
\hline Sengketa (dispute) & $\begin{array}{l}\text { Perkulakan } \\
\text { (wholesale) } \\
\text { Umo }\end{array}$ & Keuangan (finance) & Pelayan (servant) & Online (online) \\
\hline Tumpang (nearby) & $\begin{array}{l}\text { (minimum } \\
\text { wage) }\end{array}$ & Telepon (telephone) & $\begin{array}{l}\text { Penerbitan } \\
\text { (publication) }\end{array}$ & $\begin{array}{l}\text { Pegawai } \\
\text { (workers) }\end{array}$ \\
\hline $\begin{array}{l}\text { Tindih } \\
\text { (overlapping) }\end{array}$ & Job (job) & Emergency (emergency) & Proaktif (proactive) & Rotasi (rotation) \\
\hline $\begin{array}{l}\text { Implementasi } \\
\text { (implementation) }\end{array}$ & Fair (fair) & Aksesibel (accessible) & Pembuatan (make) & Negeri (country) \\
\hline Uu (laws) & $\begin{array}{l}\text { Pengembalian } \\
\text { (returning) }\end{array}$ & Tanggap (responsive) & Langsung (direct) & $\begin{array}{l}\text { Pendidik } \\
\text { (educator) }\end{array}$ \\
\hline Jabatan (position) & $\begin{array}{l}\text { Tindakan } \\
\text { (action) }\end{array}$ & Paramedik (paramedic) & Lelang (auction) & $\begin{array}{l}\text { Pengobatan } \\
\text { (treatment) }\end{array}$ \\
\hline $\begin{array}{l}\text { Kepegawaian } \\
\text { (personnel section) }\end{array}$ & $\begin{array}{l}\text { Cekungan } \\
\text { (basin) }\end{array}$ & Pemadam (extinguisher) & Mutasi (mutation) & $\begin{array}{l}\text { Sertifikasi } \\
\text { (certification) }\end{array}$ \\
\hline $\begin{array}{l}\text { Rasionalisasi } \\
\text { (rationalization) }\end{array}$ & $\begin{array}{l}\text { Otomatisasi } \\
\text { (automation) }\end{array}$ & Kebakaran (fire) & Demosi (demotion) & $\begin{array}{l}\text { Ambulans } \\
\text { (ambulance) }\end{array}$ \\
\hline Seleksi (selection) & Pintu (door) & $\operatorname{Cctv}(\operatorname{cctv})$ & Ketat (tight) & Target (target) \\
\hline Penerimaan & Ketinggian & Tawuran (communal & Pengaduan & Pelaksanaan \\
\hline
\end{tabular}




\begin{tabular}{|c|c|c|c|c|}
\hline (acceptance) & (height) & violence) & (complaining) & (implementation) \\
\hline Pejabat (officials) & Curah (pour) & Fiber (fiber) & $\begin{array}{l}\text { Pemberantasan } \\
\text { (eradication) }\end{array}$ & $\begin{array}{l}\text { Pendukung } \\
\text { (support) }\end{array}$ \\
\hline $\begin{array}{l}\text { Lhkpn (public } \\
\text { official asset report) }\end{array}$ & $\begin{array}{l}\text { Biopori } \\
\text { (biopori) }\end{array}$ & Optic (optic) & $\begin{array}{l}\text { Kpk (anti-graft } \\
\text { commission) }\end{array}$ & Hujan (rain) \\
\hline $\begin{array}{l}\text { Kejaksaan } \\
\text { (prosecutor) }\end{array}$ & Hibah (grant) & Wifi (wifi) & Kepolisian (police) & $\begin{array}{l}\text { Rth (green open } \\
\text { space) }\end{array}$ \\
\hline $\begin{array}{l}\text { Ppatk (financial } \\
\text { transaction center) }\end{array}$ & Debit (debit) & Startup (startup) & $\begin{array}{l}\text { Pembayaran } \\
\text { (payment) }\end{array}$ & $\begin{array}{l}\text { Gor (sports } \\
\text { arena) }\end{array}$ \\
\hline $\begin{array}{l}\text { Bpk (state audit } \\
\text { body) }\end{array}$ & $\begin{array}{l}\text { Intensitas } \\
\text { (intensity) }\end{array}$ & Digital (digital) & Aset (asset) & $\begin{array}{l}\text { Trotoar } \\
\text { (pedestrian) }\end{array}$ \\
\hline Auditor (auditor) & Unit (unit) & Kejuruan (vocation) & Lengkap (complete) & $\begin{array}{l}\text { Gedung } \\
\text { (building) }\end{array}$ \\
\hline $\begin{array}{l}\text { Penyisiran } \\
\text { (inspection) }\end{array}$ & $\begin{array}{l}\text { Pemindahan } \\
\text { (transfer) }\end{array}$ & Perikanan (fishery) & Sekolah (school) & Tahap (phase) \\
\hline $\begin{array}{l}\text { Pemborosan } \\
\text { (misspending) }\end{array}$ & $\begin{array}{l}\text { Konsolidasi } \\
\text { (consolidation) }\end{array}$ & Resort (resort) & Uji (test) & $\begin{array}{l}\text { Darurat } \\
\text { (emergency) }\end{array}$ \\
\hline Detail (detail) & $\begin{array}{l}\text { Gelanggang } \\
\text { (arena) }\end{array}$ & Kapal (boat) & $\begin{array}{l}\text { Kompetensi } \\
\text { (competence) }\end{array}$ & $\begin{array}{l}\text { Hiburan } \\
\text { (entertainment) }\end{array}$ \\
\hline Satuan (unit) & $\begin{array}{l}\text { Apartemen } \\
\text { (apartment) }\end{array}$ & Tua (old) & Masuk (enter) & $\begin{array}{l}\text { Panti (kind of } \\
\text { foster home) }\end{array}$ \\
\hline $\begin{array}{l}\text { Penganggaran } \\
\text { (budgeting) }\end{array}$ & Sewa (rent) & Restoran (restaurant) & Perguruan (college) & Sakit (sick) \\
\hline Sanksi (sanction) & $\begin{array}{l}\text { Pembelian } \\
\text { (purchasing) }\end{array}$ & Olahan (processed) & $\begin{array}{l}\text { Pembentukan } \\
\text { (establishment) }\end{array}$ & Rangka (frame) \\
\hline Patuh (obey) & $\begin{array}{l}\text { Penghematan } \\
\text { (saving) }\end{array}$ & Impian (dream) & $\begin{array}{l}\text { Smkn (vocational } \\
\text { school) }\end{array}$ & $\begin{array}{l}\text { Standar } \\
\text { (standard) }\end{array}$ \\
\hline Pasaran (market) & $\begin{array}{l}\text { Listrik } \\
\text { (electricity) }\end{array}$ & Walikota (city major) & Kepala (head) & Pekan (week) \\
\hline $\begin{array}{l}\text { Pencatatan } \\
\text { (recording) }\end{array}$ & $\begin{array}{l}\text { Lomba } \\
\text { (competition) }\end{array}$ & Reguler (regular) & Strategis (strategis) & $\begin{array}{l}\text { Pemda } \\
\text { (provincial } \\
\text { government) } \\
\text { Pengurusan }\end{array}$ \\
\hline Asset (asset) & Cagar (reserve) & Tema (theme) & Kampung (village) & $\begin{array}{l}\text { (handling/process } \\
\text { ing) }\end{array}$ \\
\hline $\begin{array}{l}\text { Amanat } \\
\text { (instruction) }\end{array}$ & Pegiat (activist) & Penerima (recipient) & $\begin{array}{l}\text { Rptra (child-friendly } \\
\text { public space) }\end{array}$ & Gratis (free) \\
\hline Partisipasi & Reklame & Keanggotaan & Akreditasi & Bpjs (social \\
\hline (participation) & (billboard) & (membership) & (accreditation) & security agency) \\
\hline Instansi (agency) & $\begin{array}{l}\text { Pelebaran } \\
\text { (widening) }\end{array}$ & Dinas (agency) & $\begin{array}{l}\text { Kolaborasi } \\
\text { (collaboration) }\end{array}$ & $\begin{array}{l}\text { Lurah (sub- } \\
\text { district head) }\end{array}$ \\
\hline Unggul (superior) & $\begin{array}{l}\text { Jembatan } \\
\text { (bridge) }\end{array}$ & Cabang (branch) & Space (space) & $\begin{array}{l}\text { Rsud (sub- } \\
\text { national hospital) }\end{array}$ \\
\hline Peringkat (rank) & $\begin{array}{l}\text { Penyeberangan } \\
\text { (crossing) }\end{array}$ & Retribusi (retribution) & Pelaku (actor) & Liar (wild/illegal) \\
\hline $\begin{array}{l}\text { Pemerataan (even } \\
\text { distribution) }\end{array}$ & $\begin{array}{l}\text { Multifungsi } \\
\text { (multi-function) }\end{array}$ & Klub (club) & Pasang (install) & $\begin{array}{l}\text { Kilometer } \\
\text { (kilometer) }\end{array}$ \\
\hline Iuran (retribution) & $\begin{array}{l}\text { Pengaturan } \\
\text { (arrangement) }\end{array}$ & Atlet (athlete) & $\begin{array}{l}\text { Embung (retention } \\
\text { basin) }\end{array}$ & $\begin{array}{l}\text { Kenyamanan } \\
\text { (comfort) }\end{array}$ \\
\hline $\begin{array}{l}\text { Perusahaan } \\
\text { (company) }\end{array}$ & Kabel (cable) & Kejuaraan (championship) & Rawa (swamp) & Rute (route) \\
\hline Promotif (promote) & Utilitas (utility) & Regional (regional) & Relokasi (relocation) & $\begin{array}{l}\text { Destinasi } \\
\text { (destination) }\end{array}$ \\
\hline Pasien (patient) & $\begin{array}{l}\text { Permukiman } \\
\text { (settlement) }\end{array}$ & Promotor (promoter) & Camat (district head) & $\begin{array}{l}\text { Bumd (province- } \\
\text { owned company) }\end{array}$ \\
\hline Paliatif (palliative) & $\begin{array}{l}\text { Kearifan } \\
\text { (wisdom) }\end{array}$ & Turis (tourist) & Laut (sea) & Bus (bus) \\
\hline Kategori (category) & $\begin{array}{l}\text { Perkampungan } \\
\text { (village) }\end{array}$ & Mancanegara (overseas) & $\begin{array}{l}\text { Pembatasan } \\
\text { (limitation) }\end{array}$ & \\
\hline Penyakit (disease) & $\begin{array}{l}\text { Nelayan } \\
\text { (fishermen) }\end{array}$ & Acara (event) & Laju (rate) & \\
\hline $\begin{array}{l}\text { Spesialis } \\
\text { (specialist) }\end{array}$ & $\begin{array}{l}\text { Pribadi } \\
\text { (personal) }\end{array}$ & Pagelaran (exhibition) & Penerangan (lighting) & \\
\hline
\end{tabular}




\begin{tabular}{|c|c|c|c|}
\hline $\begin{array}{l}\text { Pengendalian } \\
\text { (controlling) }\end{array}$ & $\begin{array}{l}\text { Penumpang } \\
\text { (passenger) }\end{array}$ & Kesenian (art) & Smart (smart) \\
\hline Rawat (care) & $\begin{array}{l}\text { Peremajaan } \\
\text { (rejuvenation) }\end{array}$ & Difabel (disable) & City (city) \\
\hline $\begin{array}{l}\text { Kebersihan } \\
\text { (cleanness) }\end{array}$ & $\begin{array}{l}\text { Koridor } \\
\text { (corridor) }\end{array}$ & Jompo (seniors) & $\begin{array}{l}\text { Konstruksi } \\
\text { (construction) }\end{array}$ \\
\hline Pemilik (owner) & $\begin{array}{l}\text { Lrt (light rail } \\
\text { transit) }\end{array}$ & Villa (villa) & Operator (operator) \\
\hline Kuliner (culinary) & Asian (asian) & Hotel (hotel) & Rawan (fragile) \\
\hline Aktor (actor) & Games (games) & $\begin{array}{l}\text { Pengangkutan } \\
\text { (transportation) }\end{array}$ & Integrasi (integration) \\
\hline $\begin{array}{l}\text { Persoalan } \\
\text { (problem) }\end{array}$ & $\begin{array}{l}\text { Penempatan } \\
\text { (placement) }\end{array}$ & Pengiriman (delivery) & Toilet (toilet) \\
\hline $\begin{array}{l}\text { Ketahanan } \\
\text { (resilience) }\end{array}$ & $\begin{array}{l}\text { Petugas } \\
\text { (officer) }\end{array}$ & Insinerator (incinerator) & Ruas (segment) \\
\hline Peranan (role) & $\begin{array}{l}\text { Erp (electronic } \\
\text { road pricing) }\end{array}$ & Building (building) & $\begin{array}{l}\text { Gawat } \\
\text { (extreme/danger) }\end{array}$ \\
\hline
\end{tabular}

Note: The word list was obtained from the top 300 words that have the highest word weight. The verbs are dropped from the list since they have no ideological meaning. Similar words with different suffixes also dropped and only the root words preserved (see also, Aydogan \& Slapin, 2015).

While the above word lists show meaning, and carefully consider about the significant difference of political rhetoric bias problem.

used by the candidates, an important caveat must be explained here. Some words in the document are written in different language but with similar meaning. For example, 'kewirausahaan' was mentioned by the document on the left side of policy space and 'entrepreneurship' which holds the similar meaning in Bahasa, was emerged in the document on the right side. The number of words with such case is rarely emerged in the textual data of this study. Therefore, the original text is preserved.

\section{Manifestation of policy position and rhetoric during election}

To evaluatu whether the candidate position in policy space as calculated by the Wordfish model is related to voter preferences distribution, we can use preand post-electoral survey results as proxy for voter rank-order preference. The election itself was conducted in 15 February 2017. Prior to the election date, LSI (Lingkaran Survey Indonesia) Nevertheless, it is important for the candidates in October 2016. The survey researcher to be more careful when the showed that $31,4 \%$ of the respondents textual data comprises of words from would vote for Ahok-Djarot, 21,1\% of the different language but hold similar respondents would vote for Anies-Sandi, and $19,3 \%$ respondents would vote for 
Table 4. Pre-election survey 12-14 April 2017

\begin{tabular}{lccc}
\hline & $\begin{array}{c}\text { Vote for } \\
\text { Anies-Sandi (\%) }\end{array}$ & $\begin{array}{c}\text { Vote for } \\
\text { Ahok-Djarot (\%) }\end{array}$ & $\begin{array}{c}\text { No response } \\
(\%)\end{array}$ \\
\hline Agus-Sylvy coalition of parties & 74.3 & 25.7 & 0 \\
Anies Sandi coalition of parties & 90.4 & 7.4 & 2,2 \\
Ahok-Djarot coalition of parties & 15.8 & 83.8 & 0,4 \\
Non-partisan & 31.8 & 48 & 20.3 \\
\hline
\end{tabular}

Note: Total respondents 495; margin of error $\pm 4.5 \%$.

Source: (Indikator Politik Indonesia, 2017b)

Agus-Sylvy. Meanwhile, another survey support towards Anies-Sandi rather than conducted by SMRC (Saiful Mujadi Ahok-Djarot. If the scenario is Ahok-Djarot Research Consulting) in the similar period against Agus-Sylvy, then $59,1 \%$ of Aniesshowed that around $45,4 \%$ of the Sandi supporter would deliver their vote to respondents would cast their vote to Agus-Sylvy (Kompas, 2016). These Ahok-Djarot while Agus-Sylvy was favored by $22,4 \%$ respondents and Anies-Sandi supported by $20,7 \%$ respondents (SMRC, 2016). Closer inspection to the results of both surveys hold similar pattern. Ahok-

Djarot enjoyed significant portion of support (almost half) of the respondents, while the supports for Anies-Sandi and Agus-Sylvi were nearly equal. Of course, there were various interpretations on such pattern. But one thing for sure, that there was higher potential for Anies-Sandi and Agus-Sylvi might sharing the similar supporters. And such assumption was further indicated by the results on headto-head survey scenario between candidates. The LSI survey reveals that in a head-to-head scenario of Ahok-Djarot against Anies-Sandi, around $64,3 \%$ of Agus-Sylvy supporters would divert its survey results further confirm that the voters of Agus-Sylvy, which positioned at one end of policy space above, are more inclined to cast their vote to Anies-Sandi, which hold closer policy distance towards Agus-Sylvi rather than Ahok-Djarot at the opposite end of the policy space.

The election in February 2017 resulted as follows: Ahok-Djarot (2.364.577 votes or 42,99\%); AniesSandy (2.197.333 votes or $39,95 \%)$; Agus-Sylvy (937.955 votes or $17,07 \%$ ). Since there was no majority, the second election was held in 19 April 2018 involving Ahok-Djarot and Anies-Sandi as contenders. Indikator Politik Indonesia conducted a survey from 12-14 April 2017 to map the voters preference on the two pair of candidates. The result is in table 4. 
Table 5. Exit Poll 19 April 2017

\begin{tabular}{lcc}
\hline & $\begin{array}{c}\text { Vote for } \\
\text { Anies-Sandi (\%) }\end{array}$ & $\begin{array}{c}\text { Vote for } \\
\text { Ahok-Djarot (\%) }\end{array}$ \\
\hline Agus-Sylvy coalition of parties & 81 & 19 \\
Anies Sandi coalition of parties & 94 & 6 \\
Ahok-Djarot coalition of parties & 17 & 83 \\
Non-partisan & 61 & 39 \\
\hline
\end{tabular}

Note: total respondents 798

Source: (Indikator Politik Indonesia, 2017a)

The above table shows that the we can see that political rhetoric Ahokpreference of voters with party-affiliation Djarot, which stand on the right position (Party ID) that support certain candidate of the spectrum, are levitate around shows no significant difference with the kebangsaan (nationality); penggusuran survey results conducted before the first (forced eviction); pelayanan (service); round of election. Those who support birokrasi (bureaucracy); pemborosan Agus-Sylvy were mostly devoted their vote (wasting); rasionalisasi (rationalization). to Anies-Sandi $(74,3 \%)$ rather than Ahok- And these are reflected on the candidate Djarot $(25,7 \%)$. Exit poll conducted by statement and arguments during the the similar institution on the second round campaign.

Election Day show no significant Ahok-Djarot focuses on establishment difference on the predicted survey in table of 'modern' state in Jakarta by keeping its 4.

The results of both surveys, once again, confirm that Agus-Sylvy voters felt their policy aspiration were closer to Anies-Sandi rather than Ahok-Djarot as already calculated by the Wordfish model on the candidate policy position.

To inspect the manifestation of Wordfish model result on political rhetoric from each candidate, this study observes the statement made by each candidate in the mass media and official debate during the campaign period. From table 4 above, dominant rhetoric such as keamanan current eviction (penggusuran) policy in illegally-occupied slump areas and relocating the people to governmentsponsored public housing (rusunawa) (Alsadad, 2016). In addition, the candidate also takes seriously public service, promising sort-of KPI (key performance indicator) to evaluate bureaucratic performance and salary level. From table 3, we can see that candidates on the left position i.e. Agus-Sylvy and Anies-Sandi positions are levitated around 
(security); kesenjangan (discrepancy); the poor Jakartans through the allocation penggusuran (forced eviction); so-called 'dana bergulir' program annually penindasan (exploitation); ketidakadilan (Rizki, 2017). In contrary, Ahok criticizes (injustice); kesejahteraan (welfare) and the entrepreneurship program from Anieskewirausahaan (entrepreneurship). Both Sandi, stating that such type of program is Agus-Sylvy and Anies-Sandi promise no unrealistic, citing the low rate of success forced eviction if they get elected as the from current government program for next governor for Jakarta. Agus offered stimulating entrepreneur (Mardiastuti, housing program to tackle the problem in 2016).

illegally-occupied slump areas while Anies

The first official debate of Jakarta's promoting a more 'humane'(manusiawi) approach in the relocation of citizen from slump areas (Fitrahudin, 2016; Putra, 2016). In one of his speech, Agus emotionally called that the victory of Agus-Silvy is also the victory of Jakartans poor against injustice (ketidakadilan), further mentioning that Jakarta should be led with more humane (manusiawi) way (Maulana, 2016). Another important rhetoric used by candidate on the left side of the policy spectrum was entrepreneurship (kewirausahaan). AniesSandi frequently raises the topic in their media statement and campaign event, particularly targeting youth (anak muda) as potential entrepreneur through OK-OCE (One Kecamatan, One Center for Entrepreneurship) program (Carina, 2016). Similarly, Agus-Sylvy also calls for government assistance in terms of financing small and medium business for 2017 election was held in January 2017. During the introductory session, Agus and Anies correspondingly raise the issue of security, justice, welfare, and humanity for all Jakartans. On the other hand, Ahok directly emphasizes the issue of transparency, professional, and clean bureaucracy to improve public service and human development index in Jakarta (Herlinawaty, 2017). The second debate was conducted in April 2017, involving Anies-Sandi and Ahok-Djarok as participants. When discussing about justice issue for the Jakartans, Ahok mentioned that justice related with the leader ability to avoid corruption and taking side [to one group only, emphasis added], taking into account his previous experience as non-Muslim Mayor in Belitung Timur. Meanhwile, Anies quickly pointed out to justice as the ability to resolve inequality (ketimpangan) among 
the Jakartans, between the poor and the rich, between those educated and uneducated (Aziza, 2017). Responding to Anies's statement, Ahok says that justice has been served for the poor Jakartans through Kartu Jakarta Pintar (KJP) and access for the poor to use public transportation (Transjakarta) for free. The debate, once again, show candidates' consistency on political rhetoric utilized since the onset of election campaign as specified by the Wordfish model.

\section{CONCLUSION}

Text is the great resource for political analysis since political actors produce political rhetoric to signal their political stance and preference over public issues. Electoral competition among politicians establishes diversity of policy options that can be used by the voters to maximize their utility in voting. In the election, voters decide on the basis of policy position closer to their preference on what should be done by the government once elected. This study has taking the benefit of using quantitative text analysis on the 2017 gubernatorial election in Jakarta. Using the electoral platform of candidates as data, the Wordfish model indicates two important findings.
First, the model estimates closer policy distance between Agus-Sylvy and Anies-Sandi in the policy space. Discussion on the voting preference during the election above shows that voters for both pair of candidates also more likely to share its vote each other rather than transferring their vote to AhokDjarot which stand at the opposite of the policy space. The second finding is associated with political rhetoric utilized by candidates. The Wordfish model enlisted several words associated with the pair of candidates in the left-position, i.e. Agus-Sylvy and Anies-Sandi. And this rhetoric was evident in the candidates' statement captured by the mass media and during the official debates. Likewise, Ahok-Djarot also use the political rhetoric recorded by the model for the right-side position in the policy space during the campaign and debates. Interestingly, the type of political rhetoric was mainly dominated by socio-economic issues such as forced eviction, entrepreneurship, public service, justice and inequality rather than sectarian-or identity-related rhetoric. Whether this result confirms the class-based explanation as aforementioned is beyond the objective of the study and requires further investigation. Overall, this study shows 
the greater benefit of using quantitative text analysis method to analyze programmatic politics during electoral competition in Indonesia. The recent advances in computational social science allow researcher to systematically extract information from large amounts of text with minimum constraints (temporal and financial). In the future, researcher can expand the method to study other issues such as political communication, ideology, foreign policy, legislative politics, or policy-making process in Indonesia.

\section{REFERENCES}

Adams, J., \& Meril III, S. (2005). Candidates' policy platforms and election outcomes: The three faces of policy representation. European Journal of Political Research, 44(6), 899-918.

Allen, N. W. (2015). Clientelism and the personal vote in Indonesia. Electoral Studies, 37, 73-85.

Alsadad, R. (2016, October 16). Ini Inti Visi Misi Ahok pada Pilkada DKI 2017. KOMPAS Daily. Retrieved from https://megapolitan.kompas.com/read /2016/10/06/07543021/ini.inti.visi. misi.ahok.pada.pilkada.dki.2017

Ambardi, K. (2009). Mengungkap Politik Kartel: Studi tentang Sistem
Kepartaian di Indonesia Era

Reformasi. Jakarta: Kepustakaan Populer Gramedia.

Arnold, C., Doyle, D., \& Wiesehomeier, N. (2017). Presidents, Policy Compromise and Legislative Success. Journal of Politics, 79(2), 380-395.

Aspinall, E., \& As'ad, M. U. (2016). Understanding family politics: Successes and failures of political dynasties in regional Indonesia. South East Asia Research, 24(3), 420-435. Aspinall, E., \& Sukmajati, M. (2016). Electoral Dynamics in Indonesia: Money Politics, Patronage and Clientelism at the Grassroots. (E. Aspinall \& M. Sukmajati, Eds.). Singapore: NUS Press.

Aydogan, A., \& Slapin, J. (2015). Leftright reversed: Parties and ideology in modern Turkey. Party Politics, 21(4), 615-625.

Aziza, K. S. (2017, April 13). Ini Adu Argumen Ahok dan Anies soal Keadilan untuk Warga Jakarta. KOMPAS Daily. Retrieved from https://megapolitan.kompas.com/read /2017/04/13/11204151/ini.adu.argu men.ahok.dan.anies.soal.keadilan.un tuk.warga.jakarta. \%OA

Benoit, K., \& Laver, M. (2003). Estimating Irish party policy positions 
using computer wordscoring: the 2002 election - a research note. Irish Political Studies, 18(1), 97-107.

Berenschot, W. (2018). The Political Economy of Clientelism: A Comparative Study of Indonesia's Patronage Democracy. Comparative Political Studies, 1-31.

BPS. (2017). Berita Resmi Statistik: Badan Pusat Statistik,

No. 15/02/Th.XX. Jakarta.

Carina, J. (2016, November 21). Kampanye "Rabu Bersama" AniesSandiaga Menyasar Anak Muda. KOMPAS Daily. Retrieved from https://megapolitan.kompas.com/read /2016/12/21/18452141/kampanye.r abu.bersama.aniessandiaga.menyasar.anak.muda

Choi, N. (2011). Local politics in Indonesia: Pathways to power. Abingdon: Routledge.

Erb, M., \& Sulistiyanto, P. (2009). Deepening democracy in Indonesia: Direct elections for local leaders (pilkada). Singapore: ISEAS.

Fitrahudin, A. Z. (2016, October 2). Ini Pandangan Anies Soal Penggusuran di DKI Jakarta. Detik News. Retrieved from https://news.detik.com/berita/d3311616/ini-pandangan-anies-soalpenggusuran-di-dki-jakarta
Fossati, D. (2018). A Tale of Three Cities: Electoral Accountability in Indonesian Local Politics. Journal of Contemporary Asia, 48(1), 23-49.

Hadiz, V. (2010). Localising power in post-authoritarian Indonesia: $A$ Southeast Asia perspective. Stanford: Stanford University Press.

Hamid, A. (2014). Jokowi's Populism in the 2012 Jakarta Gubernatorial Election. Journal of Current Southeast Asian Affairs, 33(1), 85-109.

Herlinawaty, R. (2017, January 13). Transkrip Debat Perdana Pilgub DKI Jakarta Segmen Satu. Tirto.id. Retrieved from https://tirto.id/transkrip-debatperdana-pilgub-dki-jakarta-segmensatu-cgXk

Indikator Politik Indonesia. (2017a). Exit Poll Pilgub DKI Jakarta Putaran Kedua 19 April 2017.

Indikator Politik Indonesia. (2017b). Peta Elektoral Pilkada DKI Jakarta Putara Kedua: Temuan Survei 12-14 April 2017.

Klingemann, H.-D., Volkens, A., Bara, J., Budge, I., \& McDonald, M. (2006). Mapping Policy Preferences //: Estimates for Parties, Electors, and Governments in Eastern Europe, European Union and OECD 1990- 
2003. Oxford: Oxford University Liddle, R. W., \& Mujani, S. (2007). Press.

Leadership, Party, and Religion:

Klinken, G. van. (2009). Patronage Explaining Voting Behavior in democracy in provincial Indonesia. In O. Törnquist, N. Webster, \& K. Stokke (Eds.), Rethinking popular representation (pp. 141-160). New York: Palgrave Macmillan.

Klüver, H. (2009). Measuring Interest Group Influence Using Quantitative Text Analysis. European Union Politics, 104), 535-549.

Kompas. (2016, October 12). Melihat Hasil Survei Pilkada DKI 2017 dari Tiga Lembaga. KOMPAS Daily.

Kompas. (2017, April 13). LBH Jakarta: Ahok Mungkin Pecahkan Rekor Penggusuran oleh Pemprov DKI. KOMPAS Daily. Retrieved from https://megapolitan. kompas.com/read /2017/04/13/13405181/lbh.jakarta. ahok.mungkin.pecahkan.rekor.pengg usuran.oleh.pemprov.dki

Laver, M. (2014). Measuring Policy Positions in Political Space. Annual Review of Political Science, 17(1), 207-223.

Laver, M., Benoit, K., \& Gary, J. (2003). Extracting Policy Positions from Political Texts Using Words as Data. American Political Science Review, 97(2), 311-331. Indonesia. Comparative Political Studies, 407), 832-857.

Lowe, W. (2015). Austin: Do Things With Words, Version 0.2.2. Retrieved from http://conjugateprior.org/software/aust in/

Mardiastuti, A. (2016). Tanggapi Program Anies-Sandiaga, Ahok: Cetak Pengusaha Enggak Gampang. Detik News. Retrieved from https://news.detik.com/berita/d3373344/tanggapi-program-aniessandiaga-ahok-cetak-pengusahaenggak-gampang

Mas'udi, W., \& Kurniawan, N. I. (2017). Programmatic Politics and Voter Preferences: The 2017 Election in Kulon Progo, Yogyakarta. Contemporary Southeast Asia, 39(3), 449-469.

Maulana, I. G. (2016, December 31). Agus Yudhoyono: 15 Februari Jadi Kemenangan Warga Atas Ketidakadilan. Detik News. Retrieved from https://news.detik.com/berita/d3385099/agus-yudhoyono-15februari-jadi-kemenangan-warga-atasketidakadilan 
Mietzner, M. (2013). Money, Power, and Ideology: Political Parties in PostAuthoritarian Indonesia. Singapore: NUS Press.

Mietzner, M., \& Muhtadi, B. (2017, May 5). Ahok's satisfied non-voters: an anatomy. New Mandala.

Proksch, S.-O., \& Slapin, J. (2010). Position Taking in European Parliament Speeches. British Journal of Political Science, 403), 587-611.

Proksch, S.-O., Slapin, J., \& Thies, M. F. (2011). Party system dynamics in post-war Japan: A quantitative content analysis of electoral pledges. Electoral Studies, 30(1), 114-124.

Putra, M. A. (2016, November 27). Agus-Silvy Janjikan Penataan Daerah Kumuh Tanpa Penggusuran. CNN Indonesia. Retrieved from https://www.cnnindonesia.com/kursip anasdki1/20161127192351-516-

175663/agus-silvy-janjikan-

penataan-daerah-kumuh-tanpapenggusuran/

Rizki, D. (2017, January 16). Agus-Sylvi Targetkan 20 Ribu Wirausaha Baru. Tribunnews. Retrieved from http://wartakota.tribunnews.com/201 7/01/16/agus-sylvi-targetkan-20-ribuwirausaha-baru
Savirani, A., \& Aspinall, E. (2017). Adversarial Linkages: The Urban Poor and Electoral Politics in Jakarta. Journal of Current Southeast Asian Affairs, 36(3), 3-34.

Schulte Nordholt, H., \& Klinken, G. van. (2007). Renegotiating boundaries: Local politics in post-Suharto Indonesia. (H. Schulte Nordholt \& G. van Klinken, Eds.). Leiden: KITLV Press.

Slapin, J., \& Proksch, S.-O. (2008). A Scaling Model for Estimating TimeSeries Party Positions from Texts. American Journal of Political Science, 52(3), 705-722.

Slater, D., \& Simmons, E. (2013). Coping by Colluding: Political Uncertainty and Promiscuous Powersharing in Indonesia and Bolivia. Comparative Political Studies, 46(11), 13661393.

SMRC. (2016). Kinerja Petahanan dan Peluang Para Penantang dalam Pilkada DKI: Temuan Survei 1-9 Oktober 2016. Jakarta.

Sobari, W. (2018). Reckoning Informal Politics: Expands the Logic of Survival and Failure of Regional Heads. Politik Indonesia: Indonesian Political Science Review, 3(1), 104-1237. 
Tomz, M., \& Van Houweling, R. P. (2008). Candidate Positioning and Voter Choice. American Political Science Review, 102(3), 303-318. Ubaid, A. H., \& Habibisubandi, H. B. (2017). Political Polarization based on Religious Identities: Empirical Evidence from the 2017 Jakarta Gubernatorial Election. Jurnal I/mu Pemerintahan, 8(4), 411-441.

Ufen, A. (2008). From "aliran" to dealignment: political parties in postSuharto Indonesia. South East Asia Research, 16(1), 5-41.

Warburton, E., \& Gammon, L. (2017, May 5). Class dismissed? Economic fairness and identity politics in Indonesia. New Mandala. Retrieved from http://www.newmandala.org/economi c-injustice-identity-politics-indonesia/ Wilson, I. (2017, April 19). Jakarta: inequality and the poverty of elite pluralism. New Mandala. 